\title{
Socket Transformation: A Feasible Alternative before Immediate Implant Placement in the Esthetic Zone
}

\author{
Li W* and Lau SL* \\ Branemark Osseointegration Centre, Hong Kong, P.R. China
}

\begin{abstract}
Front teeth situated close to buccal plate can be considered as the most challenging situation for immediate implant placement. Conventionally, implant placement will be delayed after the socket has healed up. However, if there is a high demand for esthetic and an immediate fixed restoration, socket transformation could be considered. Grafting the buccal concavity will be performed first with controlling of the infection by elective root canal and apiectomy. The extraction of problematic tooth and immediate implantation will be performed several months later after the bone graft healed up. In this sense, immediate implant will be more feasible by a thickened buccal bone in terms of implant stability and long term soft tissue esthetic. Socket transformation can make a difficult situation for immediate implantation, if not impossible, to a feasible case, thus achieving all the advantages from immediate implantation.
\end{abstract}

Keywords: Bone grafting; Immediate implant; Immediate loading; Esthetic zone

\section{Introduction}

Immediate implantation and provisionalization after extraction is always a challenge to clinicians. The advantages of immediate implant placement are well-known, especially in the esthetic zone. Other advantages include a reduction in number of surgeries, reduction in total treatment time, the preservation of alveolar bone [1-3], the maintenance of a good soft tissue profile [4] and the reduction of the patients' psychological trauma due to a lost front tooth [5]. Besides that, another important advantage is that it makes fixed immediate provisionalization possible; otherwise the patient has to wear a removable denture which will compromise both the short term and long term esthetic outcome by compression of the soft tissue around the implant. A temporary bonded bridge is sometimes possible if the occlusion allows, however it dislodges frequently.

Case selection is of utmost importance in immediate implant therapy. Lau et al. [6] published the first classification of maxillary central incisors according to the positions and angulations of the tooth roots using Cone-Beam Computer Tomogram (CBCT) for measurements. The results reflected the morphology of various kinds of sockets after extractions. The buccal and the palatal bone thickness at mid-root and apical level were compared; and their positions with reference to the mid alveolar line were defined. A classification was made with reference to these measurements. For socket position there are three types: Type B (closer to the buccal alveolar surface); Type $\mathrm{M}$ (midway between buccal and palatal alveolar surface) and Type $\mathrm{P}$ (closer to the palatal alveolar surface). By comparing the angulations of the alveolar process with the long axis of the roots, the angulations of the sockets were further classified into another three types as follows: Type 1 (root apex angulated towards palatal side or parallel to the alveolus); Type 2 (root apex angulated towards buccal side with long axis pass posterior to A point) and Type 3 (root apex angulated towards buccal side with long axis pass anterior to A point). The distribution of different types is shown in figure 1.

Type B3 tooth can be considered as the most challenging situation for immediate implant placement. They do not only have a very thin buccal plate, but the long axes of the tooth apex are angulated very buccally passing anterior to the natural concavity of the maxillary alveolar bone. If they are indicated for extraction and implant therapy, the conventional treatment will be extraction followed by implant placement after the socket has healed up. However, if there is a high
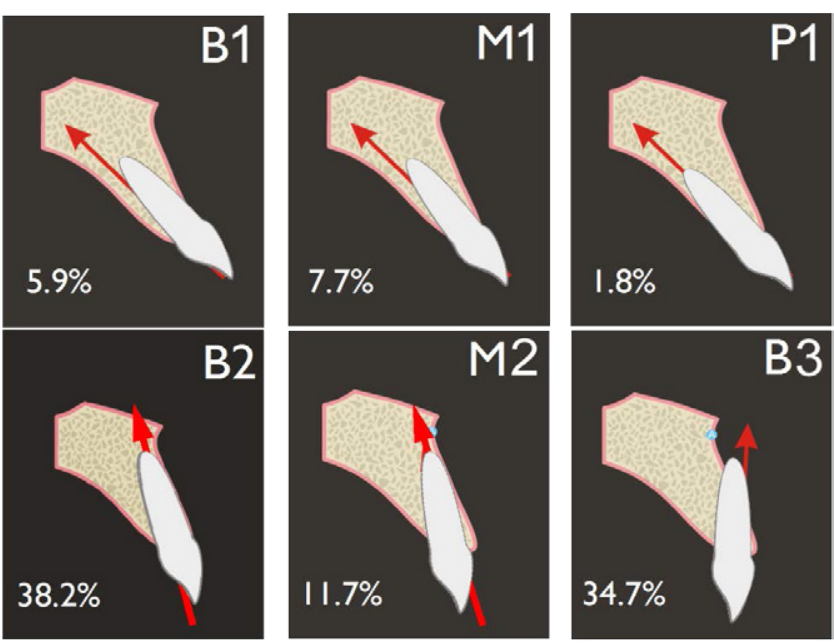

B2
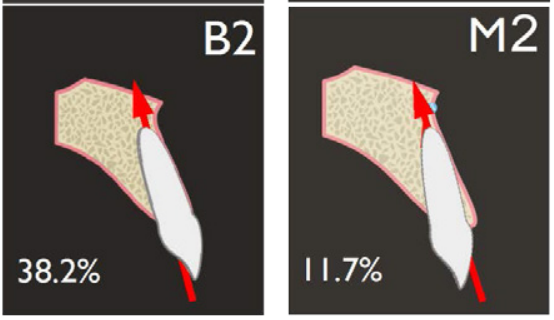

Figure 1: Classification of maxillary incisors.

demand for a long-term esthetic outcome and immediate provisional restoration, a socket transformation procedure could be considered. Grafting the buccal concavity will be performed several months ahead as the first stage, follow by the extraction of problematic tooth and immediate implantation. In this sense, the augmentation procedure will transform a Type B3 socket iatrogenically to easier type, such as Type B2. Thus immediate implant will be possible. Socket transformation can make a difficult situation for immediate implantation, if not impossible, to a feasible case, thus achieving all the advantages from immediate implantation, particularly the soft tissue profile. Two cases are reported

*Corresponding author: Li William, Branemark osseointegration centre, Room 1901, The centre, 99 Queens Road Central, Hong Kong SAR, P.R. China, Tel $852-$ 28514888. Fax 28514700, Email: William.li@disc-hk.com

Lau SL , Branemark osseointegration centre, Room 1901, The centre, 99 Queens Road Central, Hong Kong SAR, P.R. China, Tel 852-28514888. Fax 28514700, Email: fedled@hotmail.com

Received June 09, 2014; Accepted July 15, 2014; Published July 17, 2014

Citation: Li W, Lau SL (2014) Socket Transformation: A Feasible Alternative before Immediate Implant Placement in the Esthetic Zone. Dentistry 4: 248. doi:10.4172/2161-1122.1000248

Copyright: $\odot 2014 \mathrm{Li} \mathrm{W}$, et al. This is an open-access article distributed under the terms of the Creative Commons Attribution License, which permits unrestricted use, distribution, and reproduction in any medium, provided the original author and source are credited. 
Citation: Li W, Lau SL (2014) Socket Transformation: A Feasible Alternative before Immediate Implant Placement in the Esthetic Zone. Dentistry 4: 248. doi:10.4172/2161-1122.1000248

utilizing this new approach.

\section{Report of Two Cases}

\section{Patient \# 1}

A 26 year-old male experienced a trauma over his upper central incisors region at childhood. The trauma had caused both upper central incisors (\#11, \#21) to become non-vital. Root canal therapies were performed many years ago. Recurrent gingival swelling and abscess formation was noted for the last two years (Figure 2). Intra-oral radiograph showed an oblique fracture at the mid-root area of tooth \#11. CBCT showed the anterior teeth was located on the buccal side of the alveolar ridge and the corresponding root apex angulated towards the buccal side with its long axis anterior to a point (Figure 2). This is a Type B3 case according to the classification by Lau et al. [6] .

Because part of the root was found exposed anterior to the buccal bony contour and the fracture on \#11 was situated at the mid-root level, apicectomies were not considered as the definitive treatment for this condition. Dental implant replacement after conventional approach of extractions of tooth \#11 and \#21 followed by grafting procedure was discussed. However, the patient could not accept temporization using removable prosthesis and the possibilities of loss of interproximal tissue, which is well known for delayed implantation. Hence, socket transformation was suggested.

Ramus bone harvest intraorally and surgical removal of the impacted third molars on the same side were done under intra-venous sedation. There was a depression over the labial area of the anterior maxilla found intra-operatively, especially located over the apical area of tooth \#11 and $\# 21$. The two teeth were apicotomized and retrograde fillings inserted as

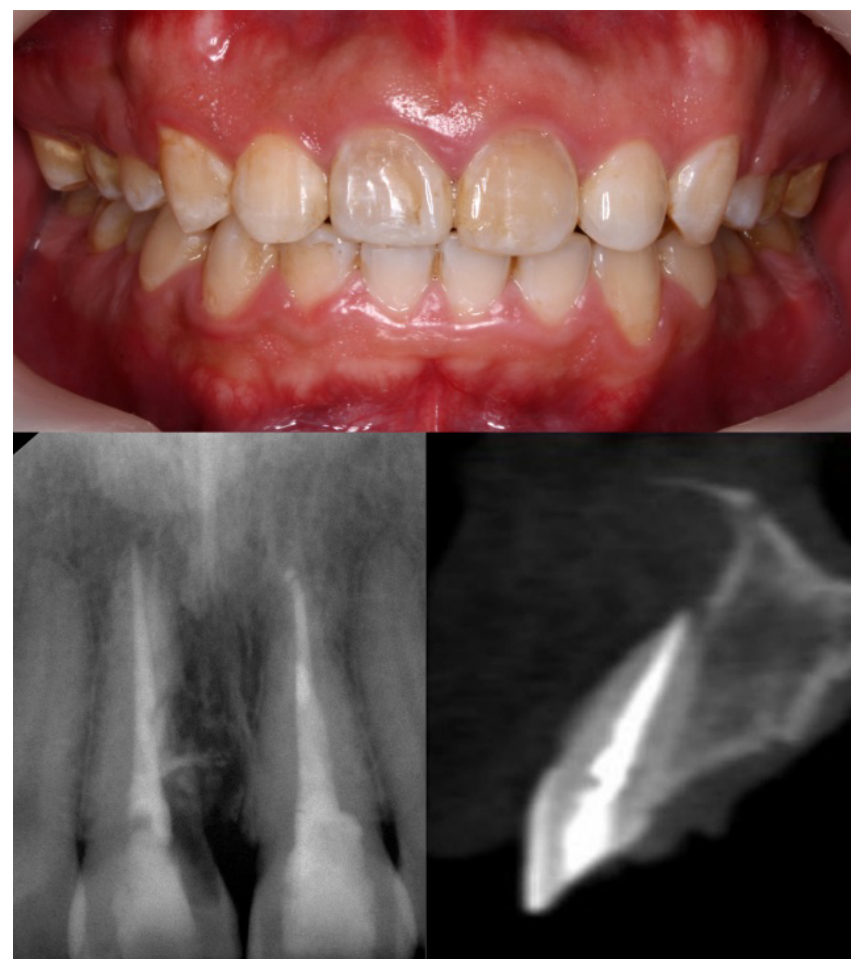

Figure 2: Pre-operative clinical photograph, radiograph and $\mathrm{CBCT}$ indicating the anterior teeth was situated on the buccal side and root apex angulated towards buccal side with long axis pass anterior to a point. Oblique fracture on \#8 was noted.

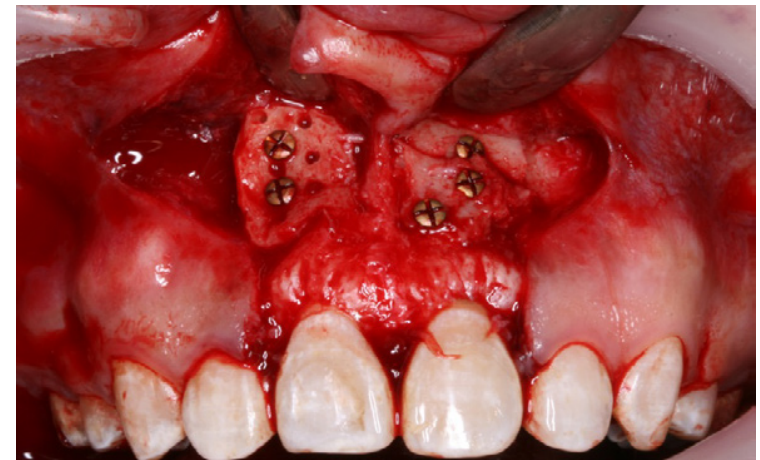

Figure 3: Bone grafting over the anterior depression by autogenous ramus bone, secured by miniscrews.

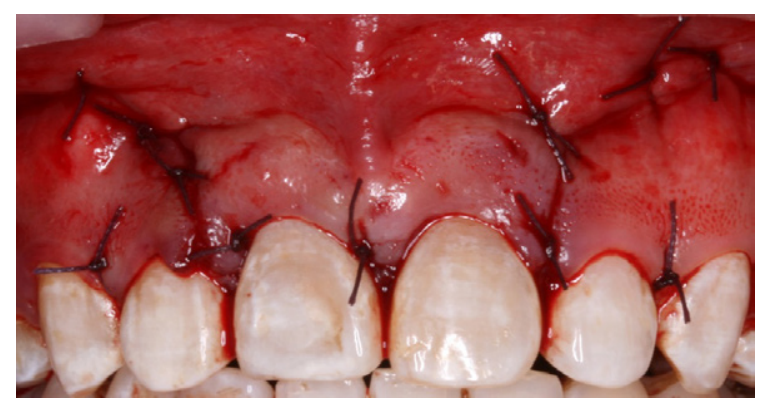

Figure 4: Immediate post-operative, keeping the existing teeth.

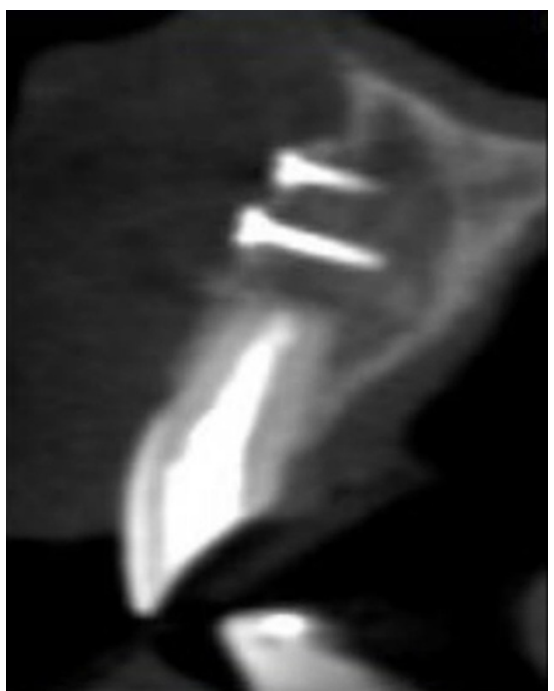

Figure 5: Post-operative $\mathrm{CBCT}$ indicating a Type B3 socket was transformed into B2.

a temporary measure to control infection. The ramus bone blocks were trimmed and stabilized to the area by titanium mini-screws (Figure 3). Nasopalatine neurovascular bundle was also removed and the canal was grafted with bone chips. The teeth were retained in the alveolar ridge while waiting for the bone graft to heal (Figure 4). Extractions with immediate implantations were performed at a later stage.

Clinical examination and further $\mathrm{CBCT}$ revealed a healed grafting site after six months. The original Type B3 socket was transformed into a Type B2 socket by thickening the buccal bone (Figure 5). Two stab 
Citation: Li W, Lau SL (2014) Socket Transformation: A Feasible Alternative before Immediate Implant Placement in the Esthetic Zone. Dentistry 4: 248. doi:10.4172/2161-1122.1000248

incisions were made for mini-screw removal, followed by extractions and immediate implantation of the two incisors. Immediate screw retained fixed provisional crown was made after implantation (Figure 6). The final zirconium abutments and crowns were constructed four months afterwards (Figure 7). It was found to be clinical stable for bone and soft tissue at 48-months follow up.

\section{Patient \#2}

A 41 year-old female was presented with missing teeth, multiple fillings and root canal therapy treated teeth at anterior maxillary region. Fixed dental prosthesis from upper right lateral incisor (\#12) to upper left lateral incisor supernumerary tooth (\#22s) was constructed many years ago. She had experienced recurrent gingival swelling, abscess and sinus tract formation at the apexes for many years. Tooth \#12 was found periodontally involved thus symptomatic, despite repeated root debridement. Tooth \# 11 and \#22s were diagnosed to have vertical

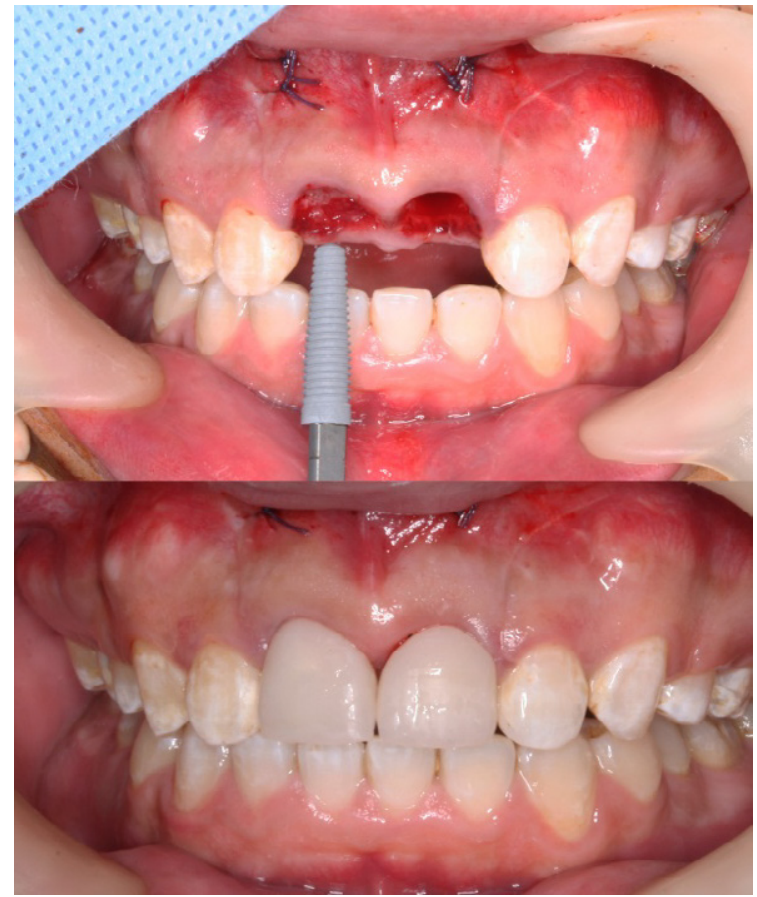

Figure 6: Immediate placement of implants after extraction of \#8 and \#9 four months after the grafting, with two stab incisions for mini-screws removal. Temporary crowns inserted immediately after the implants were placed.

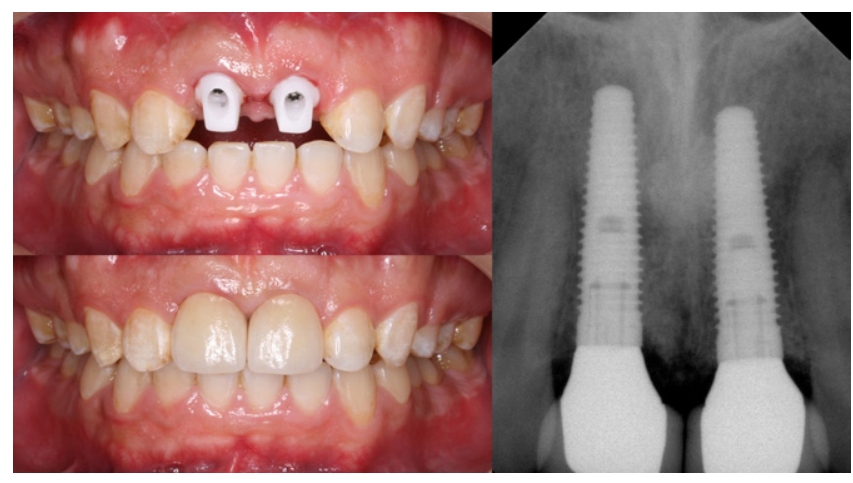

Figure 7: Final abutments and crowns inserted.

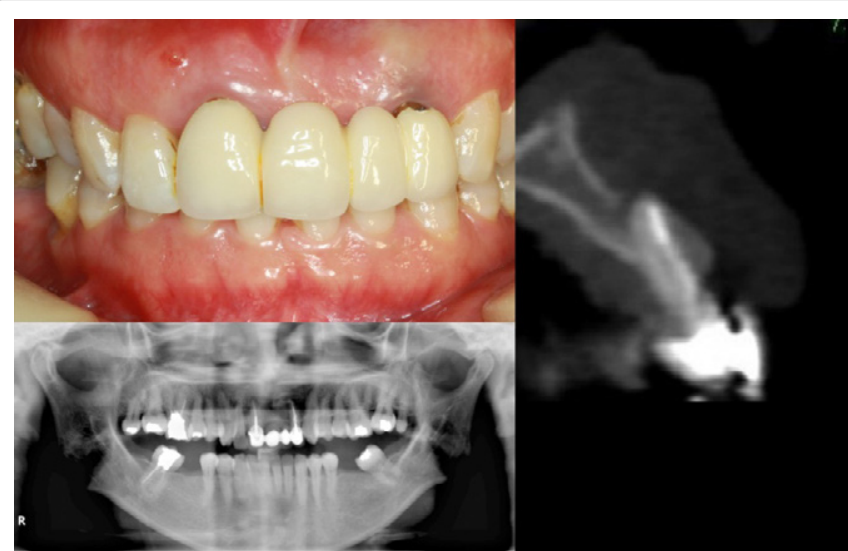

Figure 8: Pre-operative clinical photograph, OPG and CBCT indicating the teeth was situated on the buccal side and root apex angulated towards buccal side with long axis pass anterior to $A$ point. Recurrence abscesses formation between \#8 and \#7.

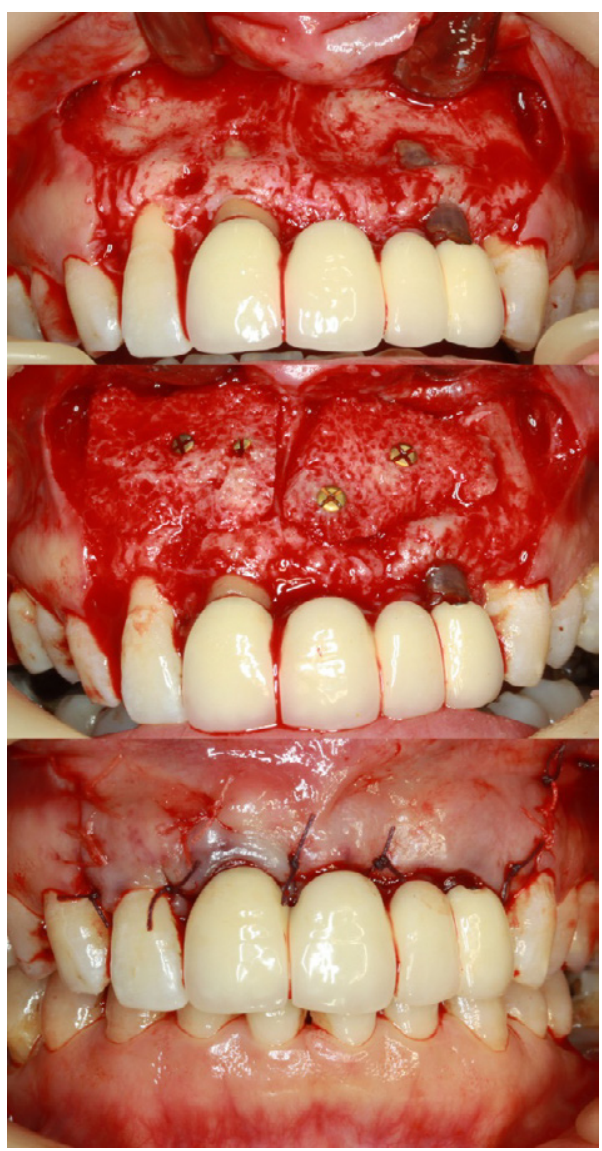

Figure 9: Bone graft over the anterior depression by Puros J-Block, secured by miniscrews after apicectomies. Immediate post-operative retaining the existing prostheses.

fractures requiring extractions. Tooth \#21 and \#22 were missing (Figure 8). Apicectomy alone would produce a non-favorable crown to root ratio for supporting a five-unit conventional bridge. Further investigation with CBCT revealed that the anterior teeth were situated on the buccal side and the corresponding root apex angulated towards the buccal plate with its long axis anterior to a point (Figure 8). This is again a Type B3 situation. Conventional approach with extractions 
of \#12, \#11 and \#22s followed by grafting procedure was discussed. However, the patient could not accept temporization using removable prosthesis. Hence socket transformation was suggested for the benefit of immediate implantation mentioned before.

A prominent depression was found located from \#12 to \#22s buccal area during the surgery. The two teeth \#11 and \#22s were apicectomized and retrograde fillings inserted as a temporary measure to control infection. We have disinfected the area and MTA was used to fill up the entire possible void so that the infection could at least be controlled temporarily. Nasopalatine neurovascular bundle was removed and the canal was grafted with bone chips. An allograft bone block (Zimmer Dental, Carlsbad) was trimmed to fit the recipient site and was stabilized by titanium mini-screws. The teeth were retained at

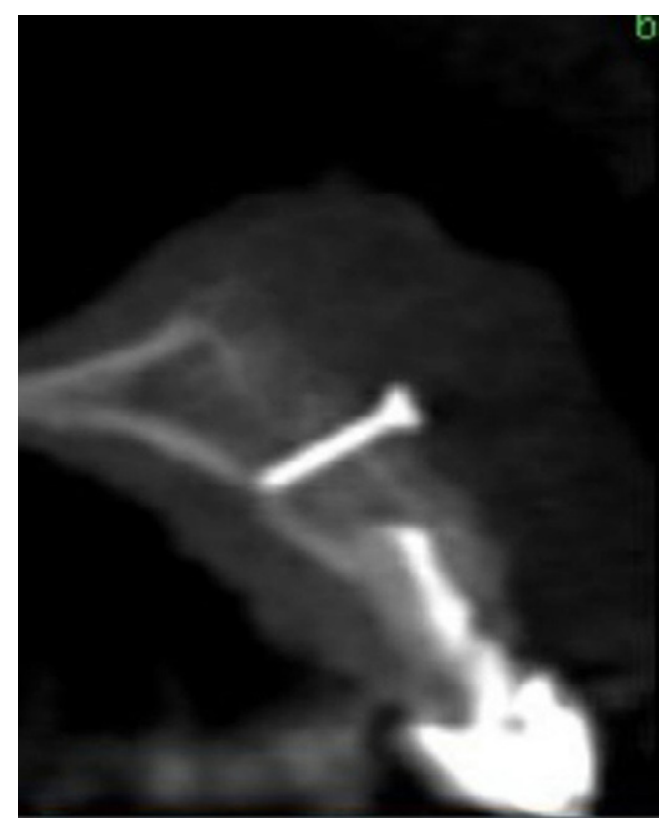

Figure 10: Post-operative CBCT indicating a Type B3 socket be transformed into an easier case.

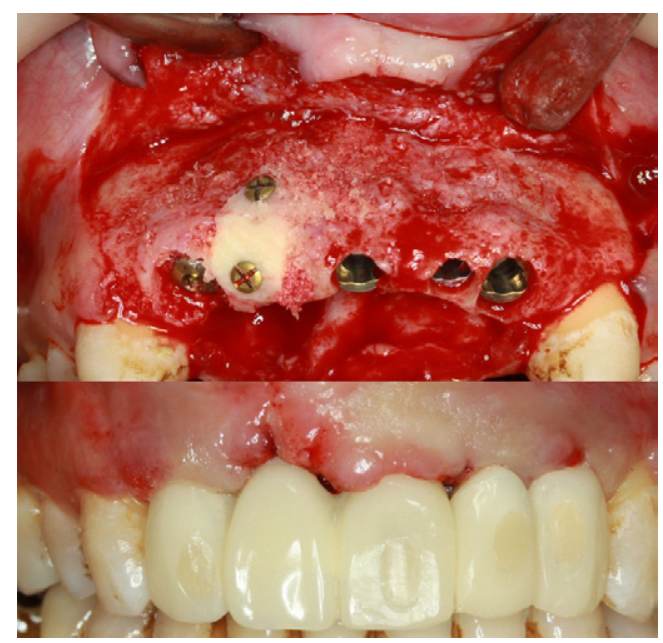

Figure 11: Placement of four implants after extraction of \#7, \#8 and \#10s, with minor graft on \#8 position. Temporary prostheses inserted immediately after the implants were placed.

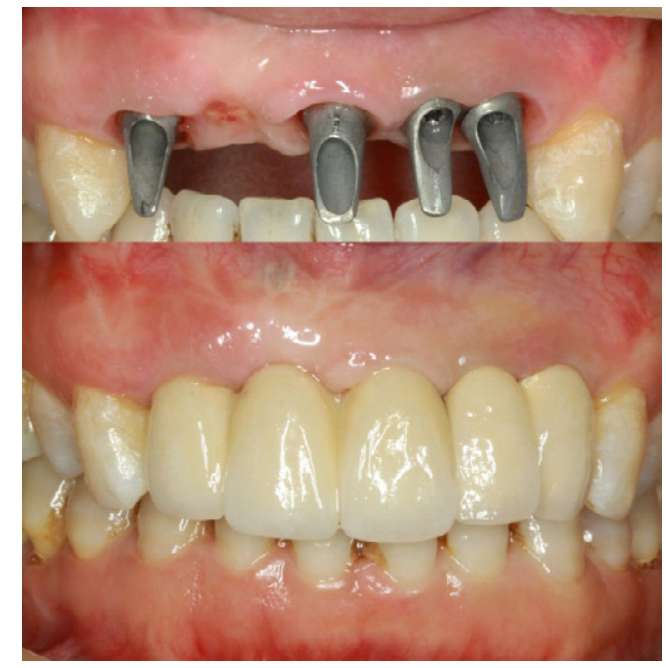

Figure 12: Final titanium abutments and prostheses inserted.

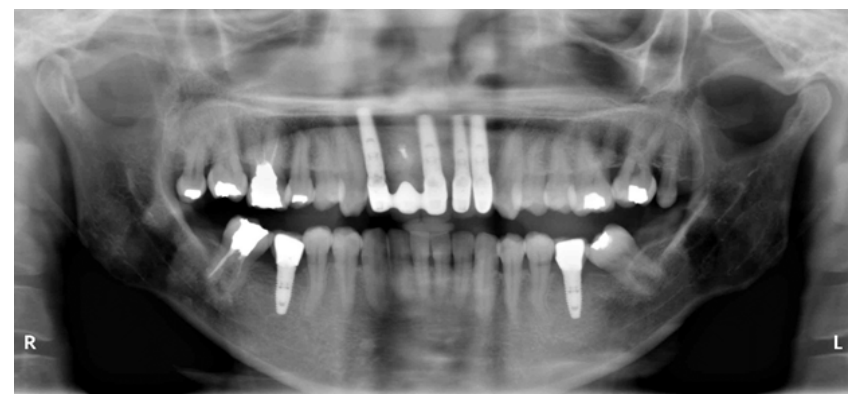

Figure 13: Post-operative radiograph.

this stage with the associated fixed prosthesis (Figure 9). Extractions with immediate implantation were performed at a later stage.

CBCT taken six months later revealed the original Type B3 condition was successfully transformed into Type B2, which was easier for immediate implantation (Figure 10). Extractions of \#12, \#11, \#22s and immediate implant placement on \#12,\#21, \#22 and \#22s were done. Additional grafting using the same allograft material was done at \#11 positions to create a better contour. Immediate provisional prosthesis was constructed and connected to the implants (Figure 11). Final titanium abutments and fixed prosthesis were installed four months afterwards (Figure 12 and 13). It was found to be clinical stable for bone and soft tissue at 38-months follow up.

\section{Discussion}

The conventional guidelines of dental implantation suggested a healing period of around three months after dental extraction of the problematic tooth, for bone to fill in and soft tissue to close up [7]. However there is always a negative effect leading to reduction in volume of both hard and soft tissue. It was reported that there is a mean reduction of bone volume of 5 to $7 \mathrm{~mm}$ buccolingually [8] and 2 to $4.5 \mathrm{~mm}$ vertically [9] during the first 4 months after dental extraction. The shrinkage of hard and soft tissue reflected a compromised esthetic outcome due to loss of interproximal tissues, recession of buccal tissue which leads to uneven gingival contouring among the anterior dentition. The effect will be exaggerated if the patient has a short lip or increase 
incisal show. The main idea of immediate implantation after extraction is to minimize all these negative effects of hard and soft tissue loss. This technique was first published in 1976 [10], after which it has become more and more popular. It has also been proven that immediate implant helps maintain a good soft tissue profile [4] thus helps achieving a good esthetic outcome. The main indication of socket transformation is to attempt transforming a difficult situation for immediate implant to a feasible one, so as to achieve the advantages of immediate implantation.

Although socket transformation can be an alternative to conventional protocol in case of high esthetic demand, or when patients demand an immediate fixed prosthesis, it is highly technique sensitive and patient cooperation is very important. The two cases reported has successfully retained the natural contour of gingival margins, which is very difficult if a conventional approach was followed. However socket transformation may not be indicated if the involved teeth are already in poor condition, such as mobility or severely periodontally involved.

Type B3 conditions like this are mostly seen on patients with maxillary alveolar hyperplasia and Angle class II div 2 occlusion. Traditionally, these cases would be managed by extracting the tooth with or without simultaneous bone grafting and inserting the implant several months later because immediate implant is deemed to be difficult. Due to the angulation of the original tooth root and its thin buccal plate, there will be a lot of difficulties if one wants to put in an implant in a position and angulation in a prosthetically friendly way. If the implant is placed in a prosthetic-driven manner, the buccal bony wall may be perforated or fenestrated. Even if the bony wall could be kept intact, there will be pressure exerted towards the already thin buccal plate, thus affecting the long-term soft tissue esthetic. Also there may be lack of primary stability of the implants for immediate provisional crown as the buccal bone is inadequate. Although sometimes a reasonable primary stability could be achieved by anchoring the implants more into the palatal or apical bone, there will still be a big difference between the angle of the implant and the prosthetic crown. If the implant is placed in this bone-driven manner, the angulation of the implant will be compromised because a reasonable out-looking prosthetic crown could not be made due to the extreme angulation. There is always a need of using an angled abutment, if the prosthetic crown is not impossible; hence the availability of such angled abutment should take into consideration before the implant is inserted. Most of the time for an abutment with a relatively big angle, there will be a step of metal on the buccal aspect. It may sometimes show through the buccal gingival margin, thus compromising the esthetic outcome. Customized abutments or ceramic abutment could also be used according to the position and angulation of the implant to reduce the esthetic problems. Placing the implant further apically may also be done to reduce the metal showing through. However, the implant position is most of the time controlled by the availability of alveolar bone.

Although there are many advantages on using socket transformation, it is not without drawbacks. The fear of getting the graft being infected is one of the most disastrous situations, as it may further compromise the already inadequate soft and hard tissue. The stability or retention of the temporary crowns or bridges may also be in doubt if the problematic tooth is in poor condition, which they cannot stand heavy occlusal forces. Although these did not happen in the above cases, further experiences and evidence are mandatory to prove it.

\section{Conclusion}

Socket transformation should be considered only for suitable cases. It could, but not always, solve the problem of long term esthetic concern for Type B3 cases. It has expanded the scope of choices for clinicians if they need immediate implants in the esthetic zone. However, further clinical studies were advised to evaluate the long-term bony and soft tissue stability for this new technique.

\section{References}

1. Shanaman $\mathrm{RH}$ (1992) The use of guided tissue regeneration to facilitate ideal prosthetic placement of implants. Int J Periodontics Restorative Dent 12: 257 265

2. Denissen HW, Kalk W, Veldhuis HA van Waas MA (1993) Anatomic consideration for preventive implantation. Int J Oral Maxillofac Implants 8: 191 196.

3. Watzek G, Haider R, Mensdorff-Pouilly N, Haas R (1995) Immediate and delayed implantation for complete restoration of the jaw following extraction of all residual teeth: a retrospective study comparing different types of serial immediate implantation. Int J Oral Maxillofac Implants 10: 561-567.

4. Werbitt MJ, Goldberg PV (1992) The immediate implant: bone preservation and bone regeneration. Int J Periodontics Restorative Dent 12: 207-217.

5. Gelb DA (1993) Immediate implant surgery: three-year retrospective evaluation of 50 consecutive cases. Int J Oral Maxillofac Implants 8: 388-399.

6. Lau SL, Chow J, Li W (2011) Classification of maxillary central incisors Implications for immediate implant in the esthetic zone. J Oral Maxillofaical Surg 69: 142-153.

7. Albrektsson T, Branematk PI, Hansson HA, Lindström J (1981) Osseointegrated titanium implants: Requirements for ensuring a long-lasting, direct bone-toimplant anchorage in man. Acta Orthop Scand 52: 155-170.

8. Johnson K (1969) A study of the dimensional changes occurring in the maxilla following tooth extraction. Aust Dent J 14: 241-244.

9. Iasella JM, Greenwell H, Miller RL, Hill M, Drisko C, et al. (2003) Ridge preservation with freeze-dried bone allograft and a collagen membrane compared to extraction alone for implant site development: a clinical and histologic study in humans. J Periodontol 74: 990-999.

10. Schulte W, Kleineikenscheidt H, Lindner K, Schareyka R (1978) [The Tubingen immediate implant in clinical studies]. Dtsch Zahnarztl Z 33: 348-359. 